TRANSACTIONS OF THE

AMERICAN MATHEMATICAL SOCIETY

Volume 361, Number 2, February 2009, Pages 889-908

S 0002-9947(08)04643-6

Article electronically published on August 15, 2008

\title{
LOCAL CHROMATIC NUMBER AND DISTINGUISHING THE STRENGTH OF TOPOLOGICAL OBSTRUCTIONS
}

\author{
GÁBOR SIMONYI, GÁBOR TARDOS, AND SINIŠA T. VREĆICA
}

\begin{abstract}
The local chromatic number of a graph $G$ is the number of colors appearing in the most colorful closed neighborhood of a vertex minimized over all proper colorings of $G$. We show that two specific topological obstructions that have the same implications for the chromatic number have different implications for the local chromatic number. These two obstructions can be formulated in terms of the homomorphism complex $\operatorname{Hom}\left(K_{2}, G\right)$ and its suspension, respectively.

These investigations follow the line of research initiated by Matoušek and Ziegler who recognized a hierarchy of the different topological expressions that can serve as lower bounds for the chromatic number of a graph.

Our results imply that the local chromatic number of 4-chromatic Kneser, Schrijver, Borsuk, and generalized Mycielski graphs is 4, and more generally, that $2 r$-chromatic versions of these graphs have local chromatic number at least $r+2$. This lower bound is tight in several cases by results of the first two authors.
\end{abstract}

\section{INTRODUCTION}

The local chromatic number is a coloring type graph parameter defined by Erdös, Füredi, Hajnal, Komjáth, Rödl, and Seress [14] in 1986. It is the number of colors appearing in the most colorful closed neighborhood of a vertex minimized over all proper colorings of the graph. Using the notation $N(v)=N_{G}(v):=\{w: v w \in$ $E(G)\}$, the formal definition is as follows.

Definition 1.1 ([14]). The local chromatic number $\psi(G)$ of a graph $G$ is

$$
\psi(G):=\min _{c} \max _{v \in V(G)}|\{c(u): u \in N(v)\}|+1,
$$

where the minimum is taken over all proper colorings $c$ of $G$.

Considering closed neighborhoods $N(v) \cup\{v\}$ results in a simpler form of the relations with other coloring parameters and explains the +1 term in the definition.

Received by the editors February 22, 2005 and, in revised form, April 16, 2007.

2000 Mathematics Subject Classification. Primary 05C15; Secondary 57M15.

Key words and phrases. Local chromatic number, box complex, Borsuk-Ulam theorem.

The first author's research was partially supported by the Hungarian Foundation for Scientific Research Grant (OTKA) Nos. T037846, T046376, AT048826, and NK62321.

The second author's research was partially supported by the NSERC grant 611470 and the Hungarian Foundation for Scientific Research Grant (OTKA) Nos. T037846, T046234, AT048826, and NK62321.

The third author's research was supported by the Serbian Ministry of Science, Grant 144026 .

(C)2008 American Mathematical Society Reverts to public domain 28 years from publication 
It is clear that $\psi(G)$ is always bounded from above by $\chi(G)$, the chromatic number of $G$. It is also easy to see that $\psi(G)=2$ is equivalent to $\chi(G)=2$. However, as is proven in [14], there exist graphs with $\psi(G)=3$ and $\chi(G)$ arbitrarily large. In this sense the local chromatic number is highly independent of the chromatic number.

On the other hand, it was observed in 26 that the fractional chromatic number $\chi_{f}(G)$ serves as a lower bound; i.e., $\chi_{f}(G) \leq \psi(G)$ holds. (For the definition and basic properties of the fractional chromatic number we refer to the books [35] and [18.) This motivated in 38 the study of the local chromatic number of graphs that have a large gap between their ordinary and fractional chromatic numbers. Basic examples of such graphs include Kneser graphs and Mycielski graphs (see [35) and their variants, the so-called Schrijver graphs (see [30, 36]) and generalized Mycielski graphs (see [19, 30], 41], 42]). Another common feature of these graphs is that their chromatic number is (or at least can be) determined by the topological method initiated by Lovász in [28]. In [38] it is proved that for all these graphs of chromatic number $t$ one has

$$
\psi(G) \geq\left\lceil\frac{t}{2}\right\rceil+1,
$$

and several cases are shown when this bound is tight. In all those cases, however, we have an odd $t$; in particular, the smallest chromatic number for which 38, gives some Schrijver graphs, say, with smaller local than ordinary chromatic number, is 5 , in spite of the fact that the lower bound $\left\lceil\frac{t}{2}\right\rceil+1$ is smaller than $t$ already for $t=4$. In this paper we show that whether $t=4$ or 5 is optimal in the above sense depends on the particular topological method that gives the chromatic number of the graph. An analogous difference between the best possible lower bound on the local chromatic number will be shown to exist for $2 r$-chromatic graphs in general. In [38] two possible topological requirements were considered that make the chromatic number of a graph at least $t$. Here we show on the one hand that since the graphs mentioned above satisfy the stronger of these two requirements, they also satisfy $\psi \geq r+2$ in the $t=2 r$ case. On the other hand, we show that the general lower bound in [38, which is derived from the weaker topological requirement considered, is tight in the sense that for all $t$ there exist graphs for which the above lower bound applies with equality. In particular, this shows that the two kinds of topological obstructions for graph coloring have different implications in terms of the local chromatic number. This consequence is in the spirit of the investigations by Matoušek and Ziegler 31] about the hierarchy they discovered among the different topological techniques bounding the chromatic number.

Some of the results (concerning the case $t=4$ ) below were announced in the brief summary [40].

\section{Preliminaries}

2.1. Topological preliminaries. The following is a brief overview of some of the topological concepts we need. We refer to [8, 20] and 30] for basic concepts and also for a more detailed discussion of the notions and facts given below. We use the notation of 30 .

A $\mathbb{Z}_{2}$-space (or involution space) is a pair $(T, \nu)$ of a topological space $T$ and the involution $\nu: T \rightarrow T$, which is continuous and satisfies that $\nu^{2}$ is the identity map. The points $x \in T$ and $\nu(x)$ are called antipodal. The involution $\nu$ and the 
$\mathbb{Z}_{2}$-space $(T, \nu)$ are free if $\nu(x) \neq x$ holds for all points $x$ of $T$. If the involution is understood from the context, we speak about $T$ rather than the pair $(T, \nu)$. This is the case, in particular, for the unit sphere $\mathbb{S}^{d}$ in $\mathbb{R}^{d+1}$ with the involution given by the central reflection $\boldsymbol{x} \mapsto-\boldsymbol{x}$. A continuous map $f: S \rightarrow T$ between $\mathbb{Z}_{2}$-spaces $(S, \nu)$ and $(T, \pi)$ is a $\mathbb{Z}_{2}$-map (or an equivariant map) if it respects the respective involutions, that is, $f \circ \nu=\pi \circ f$. If such a map exists we write $(S, \nu) \rightarrow(T, \pi)$. If $(S, \nu) \rightarrow(T, \pi)$ does not hold we write $(S, \nu) \not \rightarrow(T, \pi)$. If both $S \rightarrow T$ and $T \rightarrow S$ we call the $\mathbb{Z}_{2}$-spaces $S$ and $T \mathbb{Z}_{2}$-equivalent and write $S \leftrightarrow T$.

We sometimes refer to homotopy equivalence and $\mathbb{Z}_{2}$-homotopy equivalence (i.e., homotopy equivalence given by $\mathbb{Z}_{2}$-maps), but we will use only the following two simple observations. First, if the $\mathbb{Z}_{2}$-spaces $S$ and $T$ are $\mathbb{Z}_{2}$-homotopy equivalent, then $S \leftrightarrow T$. Second, if the space $S$ is homotopy equivalent to a sphere $\mathbb{S}^{h}$ (this relation is between topological spaces, not $\mathbb{Z}_{2}$-spaces), then $S$ is $(h-1)$-connected and therefore $\mathbb{S}^{h} \rightarrow(S, \nu)$ for any involution $\nu$; cf. [30] (proof of Proposition 5.3.2 (iv), p. 97). In the other direction we have $(S, \nu) \rightarrow \mathbb{S}^{h}$ if $(S, \nu)$ is the body of an $h$-dimensional free simplicial $\mathbb{Z}_{2}$-complex. (See below for the definition of the latter.)

The $\mathbb{Z}_{2}$-index of a $\mathbb{Z}_{2}$-space $(T, \nu)$ is defined (see e.g. 31, 30]) as

$$
\operatorname{ind}(T, \nu):=\min \left\{d \geq 0:(T, \nu) \rightarrow \mathbb{S}^{d}\right\},
$$

where $\operatorname{ind}(T, \nu)$ is set to be $\infty$ if $(T, \nu) \nrightarrow \mathbb{S}^{d}$ for all $d$.

The $\mathbb{Z}_{2}$-coindex of a $\mathbb{Z}_{2}$-space $(T, \nu)$ is defined as

$$
\operatorname{coind}(T, \nu):=\max \left\{d \geq 0: \mathbb{S}^{d} \rightarrow(T, \nu)\right\} .
$$

If such a map exists for all $d$, then we set $\operatorname{coind}(T, \nu)=\infty$. Thus, if $(T, \nu)$ is not free, we have $\operatorname{ind}(T, \nu)=\operatorname{coind}(T, \nu)=\infty$.

Note that $S \rightarrow T$ implies ind $(S) \leq \operatorname{ind}(T)$ and $\operatorname{coind}(S) \leq \operatorname{coind}(T)$. In particular, $\mathbb{Z}_{2}$-equivalent spaces have equal index and also equal coindex.

The celebrated Borsuk-Ulam Theorem can be stated in many equivalent forms. Here we state four of them. For more equivalent versions and several proofs we refer to [30. Here (i)-(iii) are all standard forms of the Borsuk-Ulam Theorem, while (iv) is clearly equivalent to (iii).

\section{Borsuk-Ulam Theorem.}

(i): For every continuous map $f: \mathbb{S}^{k} \rightarrow \mathbb{R}^{k}$ there exists $\boldsymbol{x} \in \mathbb{S}^{k}$ for which $f(\boldsymbol{x})=f(-\boldsymbol{x})$.

(ii): (Lyusternik-Schnirel'man version) Let $d \geq 0$ and let $\mathcal{H}$ be a collection of open (or closed) sets covering $\mathbb{S}^{d}$ with no $H \in \mathcal{H}$ containing a pair of antipodal points. Then $|\mathcal{H}| \geq d+2$.

(iii): $\mathbb{S}^{d+1} \nrightarrow \mathbb{S}^{d}$ for any $d \geq 0$.

(iv): For a $\mathbb{Z}_{2}$-space $T$ we have $\operatorname{ind}(T) \geq \operatorname{coind}(T)$.

The suspension $\operatorname{susp}(S)$ of a topological space $S$ is defined as the factor of the space $S \times[-1,1]$ that identifies all the points in $S \times\{-1\}$ and identifies also the points in $S \times\{1\}$. If $S$ is a $\mathbb{Z}_{2}$-space with the involution $\nu$, then the suspension $\operatorname{susp}(S)$ is also a $\mathbb{Z}_{2}$-space with the involution $(x, t) \mapsto(\nu(x),-t)$. Any $\mathbb{Z}_{2}$-map $f: S \rightarrow T$ naturally extends to a $\mathbb{Z}_{2}$-map $\operatorname{susp}(f): \operatorname{susp}(S) \rightarrow \operatorname{susp}(T)$ given by $(x, t) \mapsto(f(x), t)$. We have $\operatorname{susp}\left(\mathbb{S}^{n}\right) \cong \mathbb{S}^{n+1}$ with a $\mathbb{Z}_{2}$-homeomorphism. These observations show the well-known inequalities below. 
Lemma 2.1. For any $\mathbb{Z}_{2}$-space $S$, $\operatorname{ind}(\operatorname{susp}(S)) \leq \operatorname{ind}(S)+1$ and $\operatorname{coind}(\operatorname{susp}(S)) \geq$ $\operatorname{coind}(S)+1$.

An abstract simplicial complex $K$ is a nonempty, hereditary set system. In this paper we consider only finite simplicial complexes. The nonempty sets in $K$ are called simplices. The dimension of a $\sigma \in K$ is $\operatorname{dim}(\sigma)=|\sigma|-1$. A simplex of dimension $k$ is called a $k$-simplex. The dimension of $K$ is defined as $\max \{\operatorname{dim}(\sigma)$ : $\sigma \in K\}$. We call the set $V(K)=\{x:\{x\} \in K\}$ the set of vertices of $K$. In a geometric realization of $K$, a vertex $x$ corresponds to a point $\|x\|$ in a Euclidean space, and a simplex $\sigma$ corresponds to its body, the convex hull of its vertices: $\|\sigma\|=\operatorname{conv}(\{\|x\|: x \in \sigma\})$. We assume that the points $\|x\|$ for $x \in \sigma$ are affine independent, and so $\|\sigma\|$ is a geometric simplex. We also assume that disjoint simplices have disjoint bodies. The body of the complex $K$ is $\|K\|=\bigcup_{\sigma \in K}\|\sigma\|$. $\|K\|$ is determined up to homeomorphism by $K$. Any point in $p \in\|K\|$ has a unique representation as a convex combination $p=\sum_{x \in V(K)} \alpha_{x}\|x\|$ such that $\left\{x: \alpha_{x}>0\right\} \in K$.

A simplicial map $f: K \rightarrow L$ maps the vertices of a simplicial complex $K$ to the vertices of another simplicial complex $L$ such that the image of a simplex of $K$ is a simplex in $L$. Such a map can be linearly extended to the bodies of all simplices in $K$ giving a continuous map $\|f\|:\|K\| \rightarrow\|L\|$. A simplicial complex with a simplicial involution is called a simplicial $\mathbb{Z}_{2}$-complex.

The barycentric subdivision $s d(K)$ of a simplicial complex $K$ is the family of chains (subsets linearly ordered by inclusion) of simplices of $K$. The standard geometric realization (each simplex is represented by a point in its relative interior) gives $\|s d(K)\|=\|K\|$.

2.2. Topological lower bounds on the chromatic number. The topological method for bounding the chromatic number can be described by the following scheme. One assigns a $\mathbb{Z}_{2}$-space to all graphs in such a way that whenever a homomorphism from $F$ to $G$ exists this implies the existence of a $\mathbb{Z}_{2}$-map from the space assigned to $F$ to that assigned to $G$. Colorability with $m$ colors is equivalent to the existence of a homomorphism to $K_{m}$. If one shows that no $\mathbb{Z}_{2}$-map exists from the space assigned to $G$ to the space assigned to $K_{m}$, then it proves that $G$ is not $m$-colorable. In the cases we consider, the space assigned to $K_{m}$ will be $\mathbb{Z}_{2}$-homeomorphic to $\mathbb{S}^{f(m)}$ with $f(m)=m-2$ or $m-1$ depending on which of the two space assignments discussed below is used. Thus if $G$ is $m$-colorable, then the $\mathbb{Z}_{2}$-index of the space assigned to $G$ must not be more than $f(m)$. If it is more than $f(m)$, that implies $\chi(G)>m$. Thus we can bound the chromatic number from below by giving a lower bound on the index of a certain $\mathbb{Z}_{2}$-space. This is often done by actually bounding its coindex from below. By the Borsuk-Ulam theorem (form (iv)) this also provides a lower bound on the index.

One way to assign a $\mathbb{Z}_{2}$-space to a graph $G$ is via defining some simplicial complex, a so-called box complex, and considering the body of this complex. Following the papers [2, 27] Matoušek and Ziegler [31] defined several box complexes that turn out to fall into two categories in the sense that their index (or coindex) assumes one of only two values. (This is proven in 31, but Csorba [1] and Živaljević [46] give a further explanation of this fact by showing that the $\mathbb{Z}_{2}$-homotopy type of all these complexes is one of only two different kinds.) One representative of each of these types is given in the two definitions below. (In the second case, for simplicity, 
we speak about a cell complex and its body as the corresponding topological space. It is also $\mathbb{Z}_{2}$-homotopy equivalent to some of the known box complexes as remarked following Definition 2.3.)

For subsets $S, T \subseteq V(G)$ we denote the set $S \times\{1\} \cup T \times\{2\}$ by $S \uplus T$. For $v \in V(G)$ we denote by $+v$ the vertex $(v, 1)=\{v\} \uplus \emptyset$ and $-v$ denotes the vertex $(v, 2)=\emptyset \uplus\{v\}$.

Definition 2.2. The box complex $B_{0}(G)$ is a simplicial complex on the vertices $V(G) \times\{1,2\}$. For subsets $S, T \subseteq V(G)$ the set $S \uplus T:=S \times\{1\} \cup T \times\{2\}$ forms a simplex if and only if $S \cap T=\emptyset$ and the complete bipartite graph with sides $S$ and $T$ is a subgraph of $G$. The simplicial involution switching $+v$ and $-v$ for $v \in V(G)$ makes $B_{0}(G)$ a simplicial $\mathbb{Z}_{2}$-complex and $\left\|B_{0}(G)\right\|$ a free $\mathbb{Z}_{2}$-space.

Note that $V(G) \uplus \emptyset$ and $\emptyset \uplus V(G)$ are simplices of $B_{0}(G)$.

Definition 2.3. The hom space $H(G)$ of $G$ is the subspace of $\left\|B_{0}(G)\right\|$ consisting of those points $p \in\left\|B_{0}(G)\right\|$ that, when written as a convex combination $p=\sum_{x \in V\left(B_{0}(G)\right)} \alpha_{x}\|x\|$ with $\left\{x: \alpha_{x}>0\right\}$ being a simplex of $B_{0}(G)$, give $\sum_{x \in V(G) \uplus \emptyset} \alpha_{x}=1 / 2$. This space can also be considered as the body of a cell complex as follows. Let the hom complex $\operatorname{Hom}\left(K_{2}, G\right)$ of $G$ be the cell complex with cells $S \uplus T \in B_{0}(G)$ with $S \neq \emptyset \neq T$. We call $S \uplus T \in \operatorname{Hom}\left(K_{2}, G\right)$ a cell of the complex and $\|S \uplus T\| \cap H(G)$ is the body of this cell. The vertices of $\operatorname{Hom}\left(K_{2}, G\right)$ are of the form $\{x\} \uplus\{y\}$ with $\{x, y\} \in E(G)$.

We consider $\operatorname{Hom}\left(K_{2}, G\right)$ as a $\mathbb{Z}_{2}$-complex and $H(G)$ as a $\mathbb{Z}_{2}$-space with the involution inherited from $B_{0}(G)$.

The cell complex $\operatorname{Hom}\left(K_{2}, G\right)$ is a special case of the more general homomorphism complexes $\operatorname{Hom}(F, G)$; see [4]. The hom space $H(G)$ can also be considered as the body of a simplicial complex $B_{\text {chain }}(G)$, where $B_{\text {chain }}(G)$ is the first barycentric subdivision of $\operatorname{Hom}\left(K_{2}, G\right)$; see 31 . The latter is also $\mathbb{Z}_{2}$-homotopy equivalent to another simplicial box complex $B(G)$ (cf. [31]), where $B(G)$ is the hereditary closure of $\operatorname{Hom}\left(K_{2}, G\right)$ and it differs from $B_{0}(G)$ only by not containing those simplices $S \uplus T$ where the elements of one of the sets $S$ and $T$ do not have a common neighbor in $G$ (implying emptiness of the other set).

A useful connection between $B_{0}(G)$ and $H(G)$ follows from results of Csorba. Namely, Csorba [11] proves the $\mathbb{Z}_{2}$-homotopy equivalence of $\left\|B_{0}(G)\right\|$ and the suspension of the body of the other box complex $B(G)$ mentioned above. Further, he proves, cf. also Živaljević [46], the $\mathbb{Z}_{2}$-homotopy equivalence of $\|B(G)\|$ and $H(G)$. (A weaker version of the latter equivalence, which already implies the proposition below, also follows from the results in [31.)

Proposition $2.4([11,31,46]) \cdot\left\|B_{0}(G)\right\| \leftrightarrow \operatorname{susp}(H(G))$.

The box complex $B_{0}\left(K_{m}\right)$ is the boundary complex of the $m$-dimensional crosspolytope (i.e., the convex hull of the basis vectors and their negatives in $\mathbb{R}^{m}$ ); thus $\left\|B_{0}\left(K_{m}\right)\right\| \cong \mathbb{S}^{m-1}$ with a $\mathbb{Z}_{2}$-homeomorphism and coind $\left(\left\|B_{0}(G)\right\|\right) \leq \operatorname{ind}\left(\left\|B_{0}(G)\right\|\right)$ $\leq m-1$ is necessary for $G$ being $m$-colorable. Similarly, $\operatorname{coind}(H(G)) \leq \operatorname{ind}(H(G)) \leq$ $m-2$ is also necessary for $\chi(G) \leq m$ since $H\left(K_{m}\right)$ can be obtained from intersecting the boundary of the $m$-dimensional cross-polytope with the hyperplane $\sum x_{i}=0$, and therefore $H\left(K_{m}\right) \cong \mathbb{S}^{m-2}$ with a $\mathbb{Z}_{2}$-homeomorphism. These four lower bounds on $\chi(G)$ can be arranged in a single line of inequalities using Lemma 2.1 and 
Proposition 2.4:

$(2.1)$

$\chi(G) \geq \operatorname{ind}(H(G))+2 \geq \operatorname{ind}\left(\left\|B_{0}(G)\right\|\right)+1 \geq \operatorname{coind}\left(\left\|B_{0}(G)\right\|\right)+1 \geq \operatorname{coind}(H(G))+2$.

The first two of the lower bounds to $\chi(G)$ above are (equivalent to) the two strongest lower bounds in Matoušek and Ziegler's Hierarchy Theorem [31]. We are able to say more on the last two bounds that were singled out by the following definition in 38 .

Definition 2.5. We say that a graph $G$ is topologically $t$-chromatic if

$$
\operatorname{coind}\left(\left\|B_{0}(G)\right\|\right) \geq t-1 \text {. }
$$

We say that a graph $G$ is strongly topologically $t$-chromatic if

$$
\operatorname{coind}(H(G)) \geq t-2 \text {. }
$$

Note that if a graph is strongly topologically $t$-chromatic, then it is also topologically $t$-chromatic, and if $G$ is topologically $t$-chromatic, then $\chi(G) \geq t$.

Examples of strongly topologically $t$-chromatic graphs are provided by $t$-chromatic Kneser graphs, Schrijver graphs, and generalized Mycielski graphs. (For the formal definition of all these graphs, see, e.g., 30, or 38.) One way to show that these graphs are strongly topologically $t$-chromatic is to refer to another simplicial complex, the neighborhood complex $\mathcal{N}(G)$ of the graph $G$, introduced by Lovász in [28. Proposition 4.2 in [4] states that $\|\mathcal{N}(G)\|$ is homotopy equivalent to $H(G)$ for every graph $G$ (note that $\|\mathcal{N}(G)\|$ is not a $\mathbb{Z}_{2}$-space; thus this cannot be a $\mathbb{Z}_{2^{-}}$ homotopy equivalence). Thus if $\mathcal{N}(G)$ is homotopy equivalent to the sphere $\mathbb{S}^{t-2}$ then, by the above result in [4] and the corresponding remark in the introductory part of Subsection 2.1, we have coind $(H(G)) \geq t-2$. (In fact, since $H(G)$ is free, we have equality here.) For $t$-chromatic Schrijver graphs, Björner and de Longueville 9] proved that their neighborhood complex is homotopy equivalent to $\mathbb{S}^{t-2}$. As Schrijver graphs are induced subgraphs of Kneser graphs with the same chromatic number, this proves strong topological $t$-chromaticity for both $t$-chromatic Kneser graphs and Schrijver graphs. An analogous result about the homotopy equivalence of the neighborhood complex of $t$-chromatic generalized Mycielski graphs and $\mathbb{S}^{t-2}$ was proved by Stiebitz 41]; cf. also [19] and [30]. There is a similar result due to Lovász 29] for a finite subgraph of the Borsuk graph $B(t-1, \alpha)$ (see Definition 3.6) that we will return to in the proof of Lemma 3.7. We remark that the strong topological $t$-chromaticity of $t$-chromatic Kneser graphs and Schrijver graphs can also be seen more directly from the results of Bárány [6] and Schrijver [36]. For more details about this, cf. Proposition 8 in [38.

For examples of graphs that are topologically $t$-chromatic but not strongly topologically $t$-chromatic, we refer to the detailed discussion in Sections 4 and 5. A longer list of topologically $t$-chromatic graphs is given in 39 .

\section{LOCAL CHROMATIC NUMBER AND COVERING THE SPHERE}

In 38, the following lower bound on the local chromatic number of topologically $t$-chromatic graphs is proved.

Theorem 3.1 ([38). If $G$ is topologically $t$-chromatic for some $t \geq 2$, then

$$
\psi(G) \geq\left\lceil\frac{t}{2}\right\rceil+1 .
$$


The proof was based on an old topological theorem of Ky Fan [15] which generalizes the Borsuk-Ulam Theorem. It was also shown in [38] that this lower bound is tight for several Schrijver graphs, generalized Mycielski graphs, and Borsuk graphs of odd chromatic number.

Here we prove a similar but somewhat different lower bound than the one in Theorem 3.1. It applies only for strongly topologically $t$-chromatic graphs and gives the same conclusion if $t$ is odd; thus it is a weaker statement in that case. For $t$ even, however, the conclusion is also slightly stronger.

Theorem 3.2. If a graph $G$ is strongly topologically $t$-chromatic for $t \geq 3$, then

$$
\psi(G) \geq\left\lfloor\frac{t}{2}\right\rfloor+2 .
$$

To prove that a similar statement is not true for topologically $t$-chromatic graphs we will show in Section 4 for every $r \geq 2$ a topologically $2 r$-chromatic graph $G$ with $\psi(G)=r+1$. By Theorem 3.2 this graph cannot be strongly topologically $2 r$ chromatic. Together with Theorem 3.2 this proves that topological $t$-chromaticity and strong topological $t$-chromaticity have different implications for the local chromatic number.

First we translate the problem into one concerning open covers of the sphere.

Definition 3.3. For a nonnegative integer parameter $h$ let $Q(h)$ denote the minimum number $l$ for which $\mathbb{S}^{h}$ can be covered by open sets in such a way that no point of the sphere is contained in more than $l$ of these sets and none of the covering sets contains an antipodal pair of points.

In the earlier paper 38 , the first two authors arrived at the problem of determining $Q(h)$ through local colorings of graphs. The same question was independently asked by Micha Perles motivated by a related question of Matatyahu Rubin 1 After the publication of [38. we learned that this question was already considered and settled in papers by Ščepin [34, Izydorek, Jaworowski [21, and Jaworowski 22, 23]; cf. also Aarts and Fokkink [1. The $h=2$ case was solved even earlier by Shkliarsky 37. (The papers [34, 21]-23] use the different but equivalent formulation that we will see in Lemma 3.7 (v) below. This equivalence is already implicit in 34; cf. also [1.)

Theorem 3.4 (34, 21]-23]). For every $h \geq 1$,

$$
Q(h)=\left\lfloor\frac{h}{2}\right\rfloor+2 .
$$

Remark 3.5. The results in [38] had the implications $\left\lceil\frac{h}{2}\right\rceil+1 \leq Q(h) \leq\left\lfloor\frac{h}{2}\right\rfloor+2$, where the lower bound followed from Ky Fan's theorem [15; cf. Corollary 18 in [38. The slightly stronger Corollary 17 of [38] can also be derived from results in [1.

The relevance of the value of $Q(h)$ to local colorings will be clarified in Lemma 3.7 below. One of the conditions in the lemma uses the concept of Borsuk graphs. Their appearance in the equivalent conditions for $Q(h) \leq l$ parallels the fact that the Borsuk-Ulam theorem is equivalent to stating the chromatic number of Borsuk graphs (of appropriate parameters) as remarked by Lovász in [29].

\footnotetext{
${ }^{1}$ We are indebted to Imre Bárány [7] and Gil Kalai [24] for this information.
} 
Definition 3.6. The Borsuk graph $B(n, \alpha)$ of parameters $n$ and $0<\alpha<2$ is the infinite graph whose vertices are the points of the unit sphere in $\mathbb{R}^{n}$ (i.e., $\mathbb{S}^{n-1}$ ) and whose edges connect the pairs of points with distance at least $\alpha$.

The chromatic number of $B(n, \alpha)$ is $n+1$ for large enough $\alpha<2$ (cf. [29]). In this paper we only consider Borsuk graphs of this type; that is, when we speak about a $t$-chromatic Borsuk graph in the sequel, we always mean a $B(t-1, \alpha)$ with $\alpha<2$ large enough for making it $t$-chromatic.

Lemma 3.7. The following five statements are equivalent for every $h$ and $l$.

(i): $Q(h) \leq l$; i.e., $\mathbb{S}^{h}$ can be covered by open sets such that none of them contains an antipodal pair of points and no $\boldsymbol{x} \in \mathbb{S}^{h}$ is contained in more than $l$ of these sets.

(ii): $\mathbb{S}^{h}$ can be covered by a finite number of closed sets such that none of them contains an antipodal pair of points and no $\boldsymbol{x} \in \mathbb{S}^{h}$ is contained in more than $l$ of these sets.

(iii): There exists $0<\alpha<2$ for which $\psi(B(h+1, \alpha)) \leq l+1$.

(iv): There exists a finite graph $G$ with $\operatorname{coind}(H(G)) \geq h$ (i.e., a strongly topologically $(h+2)$-chromatic graph) such that $\psi(G) \leq l+1$.

$(\mathbf{v})$ : There is a continuous map $g$ from $\mathbb{S}^{h}$ to the body $\|K\|$ of a finite simplicial complex $K$ of dimension at most $l-1$ satisfying $g(\boldsymbol{x}) \neq g(-\boldsymbol{x})$ for all $\boldsymbol{x} \in \mathbb{S}^{h}$.

We note that, as already mentioned, the equivalence of (ii) and (v) is already implicit in 34] and is also contained partially in Lemma 5 of 1 .

We also note that for a finite graph $G$ the property $\operatorname{coind}(H(G)) \geq h$ can also be described in terms of Borsuk graphs: it is equivalent to the existence of a homomorphism from $B(h+1, \alpha)$ to $G$ for appropriately large $\alpha<2$; cf. 38.

Proof. (ii) $\Rightarrow$ (iii): Consider a covering $\mathcal{A}$ as in (ii). Consider the closed sets in the covering as colors and color each point of $\mathbb{S}^{h}$ with one of the sets containing it. We need to prove that if $\alpha<2$ is large enough, this is a proper coloring establishing $\psi(B(h+1, \alpha)) \leq l+1$.

We may assume that $|\mathcal{A}|>l$; otherwise we can add singleton sets. For each $\boldsymbol{x} \in \mathbb{S}^{h}$ let $g(\boldsymbol{x})$ be the $(l+1)$ st smallest distance of a set $A \in \mathcal{A}$ from $\boldsymbol{x}$. Since $g$ is the $(l+1)$ st level of a finite set of continuous functions, $g$ is continuous. Since $\mathbb{S}^{h}$ is compact, $g$ attains its minimum $g\left(\boldsymbol{x}_{0}\right)$. Since the covering sets are closed and $\boldsymbol{x}_{0}$ is contained in at most $l$ of them, $g\left(\boldsymbol{x}_{0}\right)>0$. For any set $A \in \mathcal{A}$ the disjoint sets $A$ and $-A$ are compact and thus they have a positive distance. Let $\delta>0$ be smaller than the minimum of $g$ and also smaller than the distance between $A$ and $-A$ for all the sets $A \in \mathcal{A}$. We choose $\alpha=\sqrt{4-\delta^{2}}$. With this choice the vertex $\boldsymbol{x}$ of $B(h+1, \alpha)$ is connected to the vertex $\boldsymbol{y}$ exactly if the distance between $\boldsymbol{y}$ and $-\boldsymbol{x}$ is at most $\delta$.

Let $\boldsymbol{x}$ be a vertex of the Borsuk graph of color $A \in \mathcal{A}$. Any vertex $\boldsymbol{y}$ connected to $\boldsymbol{x}$ is closer to $-\boldsymbol{x}$ and hence to $-A$ than $\delta$; therefore it cannot be contained in $A$. This shows that the coloring is proper.

Consider the colors of the neighbors of $\boldsymbol{x}$. These are sets with distance at most $\delta$ from $-\boldsymbol{x}$. From $g(-\boldsymbol{x})>\delta$ it follows that the number of these colors is at most $l$ as claimed. 
(iii) $\Rightarrow\left(\right.$ iv): Lovász gives in 29 a finite graph $G_{P} \subseteq B(h+1, \alpha)$ which has the property that its neighborhood complex $\mathcal{N}(G)$ is homotopy equivalent to $\mathbb{S}^{h}$. Proposition 4.2 in $[4$ states that $\mathcal{N}(F)$ is homotopy equivalent to $H(F)$ for every graph $F$; thus coind $\left(H\left(G_{P}\right)\right) \geq h$. As $G_{P} \subseteq B(h+1, \alpha)$ we have $\psi\left(G_{P}\right) \leq \psi(B(h+1, \alpha)) \leq$ $l+1$.

(iv) $\Rightarrow$ (i): Consider a proper coloring $c$ of $G$ achieving $\psi(G) \leq l+1$ and let $m$ be the number of colors used. First we give an at most $l$-fold covering of $H(G)$ by open sets $U_{1}, \ldots, U_{m}$. Let $y \in H(G)$ and let $Z_{y} \uplus T_{y}$ be the minimal cell of $\operatorname{Hom}\left(K_{2}, G\right)$ (or equivalently, the minimal simplex of $B_{0}(G)$ ) whose body contains $y$. We let $y$ belong to $U_{i}$ if and only if there is some vertex $v \in Z_{y}$ for which $c(v)=i$. It is clear that the sets $U_{i}$ obtained this way are open. As $Z_{y} \neq \emptyset$ the point $y$ is covered by some $U_{i}$. As $T_{y}$ is not empty, we can choose a vertex $w \in T_{y}$. All vertices $v \in Z_{y}$ are neighbors of $w$, so by the definition of $\psi(G)$ these vertices have at most $l$ different colors. Therefore $y$ is covered by at most $l$ sets $U_{i}$. The sets $U_{i}$ therefore form an at most $l$-fold covering of $H(G)$. For antipodal points $y, y^{\prime} \in H(G)$ we have $Z_{y^{\prime}}=T_{y}$. If $y$ and $y^{\prime}$ are contained in the same set $U_{i}$, then we find vertices $v \in Z_{y}$ and $w \in T_{y}$ of the same color $i$. As $v$ and $w$ are adjacent and $c$ is a proper coloring this is impossible, so the sets $U_{i}$ contain no antipodal pairs of points.

By the condition coind $(H(G)) \geq h$ there is a $\mathbb{Z}_{2}$-map $f: \mathbb{S}^{h} \rightarrow H(G)$. Now we define $A_{i}:=\left\{\boldsymbol{x} \in \mathbb{S}^{h}: f(\boldsymbol{x}) \in U_{i}\right\}$. It is straightforward that the open sets $A_{1}, \ldots, A_{m}$ provide a required covering.

(i) $\Rightarrow(\mathrm{v})$ : Assume that (i) holds. As $\mathbb{S}^{h}$ is compact we can assume that the open cover is finite; it consists of the sets $A_{1}, \ldots, A_{m}$. Let $K$ be the simplicial complex having vertices $[m]=\{1, \ldots, m\}$ and all $l$-subsets of $[m]$ as maximal simplices. Define $g: \mathbb{S}^{h} \rightarrow\|K\|$ as follows. Let $d_{i}(\boldsymbol{x})$ be the distance of $\boldsymbol{x} \in \mathbb{S}^{h}$ from $\mathbb{S}^{h} \backslash A_{i}$. Note that $d_{i}(\boldsymbol{x})>0$ if and only if $\boldsymbol{x} \in A_{i}$. We normalize $d_{i}$ to get $\alpha_{i}(\boldsymbol{x})=d_{i}(\boldsymbol{x}) /\left(\sum_{j=1}^{m} d_{j}(\boldsymbol{x})\right)$. Now set $g(\boldsymbol{x})$ to be the formal convex combination of the vertices of $K$ given by $\sum_{i=1}^{m} \alpha_{i}(\boldsymbol{x})\|i\|$. Since no $\boldsymbol{x} \in \mathbb{S}^{h}$ is covered by more than $l$ of the sets $A_{i}$, the images are indeed in $\|K\|$. As the sets $A_{i}$ do not contain antipodal points we have $g(\boldsymbol{x}) \neq g(-\boldsymbol{x})$; furthermore the minimal simplices containing $g(\boldsymbol{x})$ and $g(-\boldsymbol{x})$ are disjoint.

(v) $\Rightarrow$ (ii): Let $g$ be a map as in (v). We assume that the minimal simplices containing $g(\boldsymbol{x})$ and $g(-\boldsymbol{x})$ are disjoint for every point $\boldsymbol{x} \in \mathbb{S}^{h}$. If this condition is violated we consider an arbitrary geometric realization of $K$ and the continuous function $\boldsymbol{x} \mapsto \operatorname{dist}(g(\boldsymbol{x}), g(-\boldsymbol{x}))>0$. As $\mathbb{S}^{h}$ is compact this continuous function has a minimum $\varepsilon>0$. Now take an iterated barycentric subdivision $s d^{t}(K)$ of $K$ with the standard geometric realization $\left\|s d^{t}(K)\right\|=\|K\|$. As the dimension of $s d^{t}(K)$ is the same as that of $K$, we can simply consider $s d^{t}(K)$ with the same map $g: \mathbb{S}^{h} \rightarrow\left\|s d^{t}(K)\right\|$. If $t$ is high enough the maximum diameter of the body of a simplex in $s d^{t}(K)$ is below $\varepsilon / 2$ and therefore our assumption on antipodal points is satisfied.

Let the set of vertices of $K$ be $[m]=\{1, \ldots, m\}$. We define $A_{i} \subseteq \mathbb{S}^{h}$ for all $i$ in $[m]$ by letting $\boldsymbol{x} \in A_{i}$ if and only if $\alpha_{i}=\max _{j} \alpha_{j}$ in the formal convex combination $g(\boldsymbol{x})=\sum_{j=1}^{m} \alpha_{j}\|j\|$ with $\left\{j: \alpha_{j}>0\right\} \in K$. Clearly, the closed sets $A_{i}$ cover $\mathbb{S}^{h}$. As $\boldsymbol{x} \in A_{i}$ implies that $i$ is a vertex of the minimal simplex containing $g(\boldsymbol{x})$ the point $\boldsymbol{x}$ is contained in at most $l$ of the sets $A_{i}$, and by our assumption above no set $A_{i}$ contains antipodal pairs of points. 
The following corollary is just a restatement of the implication (iv) $\Rightarrow$ (i) of the above lemma for later reference.

Corollary 3.8. For any finite graph $G$ we have $Q(\operatorname{coind}(H(G))) \leq \psi(G)-1$.

Remark 3.9. Using the fact that any $d$-dimensional simplicial complex has a geometric realization in $\mathbb{R}^{2 d+1}$ (cf. Theorem 1.6.1 in [30]) and Lemma 3.7, one can show that $Q(h) \geq\left\lceil\frac{h}{2}\right\rceil+1$, i.e., the same lower bound that Ky Fan's theorem implied in 38. Indeed, by Lemma 3.7. $Q(h) \leq\left\lfloor\frac{h-1}{2}\right\rfloor+1$ would imply the existence of a continuous map $g: \mathbb{S}^{h} \rightarrow\|K\|$ where $K$ is an at most $\left\lfloor\frac{h-1}{2}\right\rfloor$-dimensional simplicial complex and $g(\boldsymbol{x}) \neq g(-\boldsymbol{x})$ for any $\boldsymbol{x} \in \mathbb{S}^{h}$. But $K$ can be realized in $\mathbb{R}^{h}$, so this way we would obtain a continuous map from $\mathbb{S}^{h}$ to $\mathbb{R}^{h}$ with no coinciding images of antipodal points. This would contradict the Borsuk-Ulam theorem.

Using the fact that the universal cover of a connected 1-dimensional complex is a (usually infinite) tree, which can be embedded in $\mathbb{R}^{2}$, the above argument can be extended to prove Shkliarsky's result [37] stating $Q(2) \geq 3$. This method, however, fails to show $Q(2 r)>r+1$ for $r>1$, which is the most difficult statement in the lower bound part of Theorem 3.4, cf. [34, 21]-23].

Note that the lower bound $\left\lceil\frac{h}{2}\right\rceil+1 \leq Q(h)$ implied by Ky Fan's theorem (cf. [38) together with Corollary 3.8 readily implies a weaker version of Theorem 3.1 . Namely, they imply that if $G$ is strongly topologically $t$-chromatic for some $t \geq 2$, then $\psi(G) \geq\lceil t / 2\rceil+1$.

Proof of Theorem 3.2, By Corollary 3.8 we have $Q(\operatorname{coind}(H(G))) \leq \psi(G)-1$. Using Theorem 3.4 this implies $\psi(G) \geq\left\lfloor\frac{t}{2}\right\rfloor+2$ if $\operatorname{coind}(H(G))=t-2 \geq 1$.

Thus any $t$-chromatic Kneser graph, Schrijver graph, generalized Mycielski graph, or Borsuk graph has local chromatic number at least $\lfloor t / 2\rfloor+2$. For Borsuk graphs it follows immediately from Lemma 3.7 that this bound is sharp for $B(t-1, \alpha)$ if $\alpha<2$ is large enough. The results in 38. imply that it is also sharp for many Schrijver graphs and generalized Mycielski graphs. This was shown there for odd $t$, while for even $t$ a gap of 1 remained in 38 (compared to the lower bound proven there). This gap is closed now. Thus we can formulate the following corollary generalizing Theorems 3 and 5 of 38 for the even chromatic case. For the precise meaning of the phrase "defining parameters" in the statement below we refer the reader to the corresponding cited statements of [38.

Corollary 3.10. Let $t$ be fixed. If $G$ is a $t$-chromatic Schrijver graph or a $t$ chromatic generalized Mycielski graph with large enough defining parameters, then

$$
\psi(G)=\left\lfloor\frac{t}{2}\right\rfloor+2 .
$$

Proof. The lower bound follows from Theorem 3.2 and the fact that these graphs are strongly topologically $t$-chromatic. The matching upper bound follows from Theorems 3 and 5 in 38.

The upper bound is trivial when $t=4$; thus there we have, unconditionally, that any 4-chromatic Kneser graph, Schrijver graph, generalized Mycielski graph, or Borsuk graph has local chromatic number 4. 
Remark 3.11. Let the graph $G$ be a quadrangulation of a compact two dimensional surface $R$; i.e., $G$ is drawn in the surface with all the resulting cells being quadrangles. In this case $H(G)$ is closely related to $R$. In particular it is easy to show that $\operatorname{coind}(H(G)) \geq 2$ if $G$ is a quadrangulation of the projective plane and $G$ is not bipartite. Using Theorem 3.2 this implies that the local chromatic number of $G$ is at least 4 generalizing the lower bound part of Youngs' result [45] which states that such graphs are 4-chromatic. It has been widely studied when quadrangulations of surfaces have (ordinary) chromatic number at least 4; see [3, 32, 45]. In such cases four distinct colors can always be found locally: any proper coloring has a multicolored quadrangular cell. (We call a set of vertices or a subgraph multicolored if every vertex in it receives a different color.) Thinking of this four-cycle as a complete bipartite graph there is a clear connection to what is called the Zig-zag Theorem in 38. (cf. also Ky Fan's paper [16]). Proving that the local chromatic number is at least 4 constitutes finding a different multicolored subgraph: a star with four vertices. This seems to be harder. The observation that nonbipartite quadrangulations of the projective plane have local chromatic number at least 4 generalizes to certain quadrangulations of the Klein bottle. Surprisingly, there are quadrangulations of other surfaces for which a multicolored cell can be found in every proper coloring but the local chromatic number is only 3. See the forthcoming paper [33] on quadrangulations of surfaces.

In view of the results in 38 and 39] it seems natural to ask what complete bipartite graphs $K_{k, l}$ must have a multicolored copy in every proper coloring of any (strongly) topologically $t$-chromatic graph. To avoid trivialities we always assume $k, l \geq 1$ when speaking about $K_{k, l}$. Using the results in 38] and this paper we can give a complete answer for topologically $t$-chromatic graphs and an almost complete answer for strongly topologically $t$-chromatic graphs. Note that 39 treated the same problem for proper $t$-colorings of topologically $t$-chromatic graphs and found a different characterization.

Let us consider topologically $t$-chromatic graphs first. As some of these graphs are indeed $t$-chromatic we must have $k+l \leq t$. By Corollary 4.9 (see below in Section (4) the local chromatic number of some of them is $\lceil t / 2\rceil+1$, so we must also have $k, l \leq\lceil t / 2\rceil$. For the remaining graphs $K_{k, l}$, the Zig-zag Theorem of [38] provides a positive answer: any proper coloring of a topologically $t$-chromatic graph contains a multicolored copy of $K_{\lfloor t / 2\rfloor,\lceil t / 2\rceil}$ and thus also of its subgraphs.

A $t$-coloring of a topologically $t$-chromatic graph cannot avoid a multicolored copy of $K_{k, l}$ for any pair of natural numbers $k, l$ with $k+l \leq t$ [39], but some $(t+1)$ colorings simultaneously avoid multicolored copies of all graphs $K_{k, l} \nsubseteq K_{\lfloor t / 2\rfloor,[t / 2\rceil}$. For even $t$ this follows from Corollary [4.9, while for odd $t$ this is stated in [38]. (Notice that in this latter case we need to avoid multicolored $K_{\lceil t / 2\rceil,[t / 2\rceil}$ subgraphs, which does not follow from attaining local chromatic number $\lceil t / 2\rceil+1$.)

Some strongly topologically $t$-chromatic graphs are also $t$-chromatic but as we have seen their lowest possible local chromatic number is $\lfloor t / 2\rfloor+2$ (attained by some Schrijver, Borsuk and generalized Mycielski graphs; see [38). This means that in order to always find a multicolored copy of $K_{k, l}$ in a proper coloring of a strongly topologically $t$-chromatic graph we need $k+l \leq t$ and $k, l \leq\lfloor t / 2\rfloor+1$. Similarly to the previous case, a single $(t+1)$-coloring of a strongly topologically $t$-chromatic graph can avoid multicolored copies of $K_{k, l}$ for all $k, l$ with $k+l>t$ or $\max (k, l)>\lfloor t / 2\rfloor+1$. For $t$ odd this is proven in [38]. It easily extends also 
to the even $t$ case by taking the Mycielskian $M(G)$ of a strongly topologically $(t-1)$-chromatic graph $G$ with a coloring of the above type and extending this to a proper coloring of $M(G)$ (which is a strongly topologically $t$-chromatic graph) in the following way. We keep the original coloring in the first layer, introduce a new color for all of the second layer and use one of the old colors for the top vertex. (For the definition of Mycielskians we refer again to [35] or [38. In the latter paper $M(G)$ is also denoted by $M_{2}(G)$.)

For most of the remaining complete bipartite graphs the Zig-zag Theorem implies the existence of a multicolored version; the only case not covered is that of $K_{t / 2+1, l}$ for even $t$ and $1 \leq l \leq t / 2-1$. Theorem 3.2 is equivalent to an affirmative answer in the $l=1$ case. For $l>1$ we do not know the answer. Here we ask the problem in the strongest possible form corresponding to $l=t / 2-1$

Question. Let $t \geq 6$ be an even integer. Is it true that if a strongly topologically $t$-chromatic graph is properly colored (with any number of colors), then it always contains a multicolored $K_{t / 2-1, t / 2+1}$ subgraph?

Using similar techniques to those used in [38 and [39] and also in this paper, an affirmative answer would immediately follow from an affirmative answer to the following topological analog of the above question.

Topological question. Let $h \geq 4$ be even and let the sphere $\mathbb{S}^{h}$ be covered by open sets $A_{1}, \ldots, A_{m}$ that satisfy $A_{i} \cap\left(-A_{i}\right)=\emptyset$ for all $i$. Is it true that there always exists an $x \in \mathbb{S}^{h}$ such that $x$ is covered by at least $h / 2+2$ and $-x$ is covered by at least $h / 2$ different $A_{i}$ 's?

Note that an affirmative answer would give a strengthening of the lower bound part of Theorem 3.4. while the two are equivalent if we set $h=2$. If $m=h+2$, then the statement is a (very) special case of the Tucker-Bacon Theorem (cf. [5, 43]), which was applied in 39 .

\section{TOPOLOGICAL $t$-CHROMATICITY VERSUS STRONG TOPOLOGICAL $t$-CHROMATICITY}

In this section we compare topological $t$-chromaticity and strong topological $t$ chromaticity, especially in their implications to the local chromatic number.

As stated in (2.1) strong topological $t$-chromaticity implies topological $t$-chromaticity, which, in turn, implies that the graph is indeed at least $t$-chromatic. It is easy to see that for $t=2$ or 3 both topological conditions are equivalent with the graph having chromatic number at least $t$. This is not the case for $t \geq 4$ as follows from an observation by Walker [44] made also by Matoušek and Ziegler [31. This observation (in terms of [31]) is that any graph $G$ without a 4 -cycle satisfies $\operatorname{ind}(\|B(G)\|) \leq 1$. Using the already mentioned $\mathbb{Z}_{2}$-homotopy equivalence of $H(G)$ and $\|B(G)\|$ and the result of Erdős 13 that there exist graphs with arbitrarily high chromatic number and girth this shows that the two sides of the first inequality in (2.1) can be arbitrarily far apart.

If one of the other three inequalities in (2.1) is strict, then we have ind $(H(G))>$ coind $(H(G))$. $\mathbb{Z}_{2}$-spaces having different index and coindex are called nontidy by Matoušek [30. Constructing such spaces does not seem obvious, but such constructions are known; see, e.g., a list in [30, page 100. Csorba [1] and Živaljević [46] proved that for any finite free $\mathbb{Z}_{2}$-complex $K$ there exists a finite graph $G$ such that $\|B(G)\|$ (and thus also $H(G)$ ) is $\mathbb{Z}_{2}$-homotopy equivalent to $\|K\|$. Some of the 
nontidy spaces, e.g., the projective space $\mathbb{R} P^{2 i-1}$ with a suitable involution, have a triangulation (i.e., it is $\mathbb{Z}_{2}$-homeomorphic to the body of a finite $\mathbb{Z}_{2}$-complex). So we have examples of graphs $G$ with $\operatorname{ind}(H(G))>\operatorname{coind}(H(G)$ ), and (from the properties of $\left.\mathbb{R} P^{2 i-1}\right)$ even coind $(H(G))=1$ with ind $(H(G))$ arbitrarily high. This shows that the difference between the two sides of at least one of the second, third, or last inequalities of (2.1) is unbounded (but, as mentioned below, it certainly cannot be the second). Further study of the $\operatorname{space} \operatorname{susp}\left(\mathbb{R}^{2 i-1}\right)$ shows that its coindex is 2 [47, 17, showing that for the above graphs there is an unbounded difference between the two sides of the third inequality in (2.1), while the last inequality holds with equality.

Based on another example appearing in [30, page 100, constructed by Csorba, Matoušek, and Živaljević, an example of a $\mathbb{Z}_{2}$-space $X$ is demonstrated by Csorba 11] which satisfies $\operatorname{ind}(X)=\operatorname{ind}(\operatorname{susp}(X))$. Since this space can also be triangulated, it shows the existence of graphs for which the second inequality is strict in (2.1) (using again the above mentioned result of Csorba [11] and Živaljević [46]). Nevertheless, as $H(G)$ is contained in $B_{0}(G)$ the sides of the second inequality can differ by at most 1 .

Our main concern is the last inequality of (2.1), which is between the defining quantities of topological and strongly topological $t$-chromaticity. Here we show not only the possibility of strict inequality, but also the existence of a topologically $t$ chromatic but not strongly topologically $t$-chromatic graph for which Theorem 3.1 is tight while $t$ is even. (For odd $t$ several examples are shown in 38 for the tightness of the lower bound in Theorem [3.1 however, those examples are also strongly topologically $t$-chromatic.) In the case of $t=2 r$ this means that our graph has local chromatic number $r+1$ in contrast to strongly topologically $2 r$-chromatic graphs for which the local chromatic number must be at least $r+2$ according to Theorem 3.2 . Thus our examples will not only separate topological $2 r$-chromaticity from strong topological $2 r$-chromaticity but show that the difference is in fact relevant also in terms of its consequences for the local chromatic number. We do not have examples where the sides of the last inequality of (2.1) differ by more than 1 .

Our examples of topologically $t$-chromatic graphs with local chromatic number equal to $\left[\frac{t}{2}\right]+1$, the lower bound in Theorem 3.1 are the universal graphs $U(2 r-1, r)$ defined below in the more general setting as they appear in [14. From now on we keep using the notation $[m]=\{1, \ldots, m\}$ already introduced in the proof of Lemma 3.7

Definition 4.1 ([14). For positive integers $r \leq m$ we define the graph $U(m, r)$ as follows:

$$
\begin{aligned}
& V(U(m, r))=\{(i, A): i \in[m], A \subseteq[m],|A|=r-1, i \notin A\} \\
& E(U(m, r))=\{\{(i, A),(j, B)\}: i \in B, j \in A\} .
\end{aligned}
$$

The graphs $U(m, r)$ characterize local chromaticity in the sense that a graph $G$ satisfies $\psi(G) \leq r$, and this value can be attained by a coloring with at most $m$ colors, if and only if there is a homomorphism from $G$ to $U(m, r)$ (see Lemma 1.1 in [14]). In particular, it is easy to find the coloring showing $\psi(U(m, r)) \leq r$ : for each vertex $(i, A)$ use $i$ as its color. We refer to this coloring as the natural coloring of $U(m, r)$. (Note that $\chi(U(m, r))<m$ whenever $m>r$, cf. [14; thus this is not an optimal coloring concerning the number of colors used. In fact, it is easy to see that if $\psi(G)<\chi(G)$, then any coloring of $G$ attaining $\psi(G)$ must use more than 
$\chi(G)$ colors. The reason is that in a proper coloring of $G$ with $\chi(G)$ colors each color class must contain a vertex which has a neighbor in all other color classes. Otherwise the color class with no such vertex could be eliminated, resulting in a proper coloring with less than $\chi(G)$ colors.)

Remark 4.2. The above discussion shows that the local chromatic number fits into the framework described in Chapter 1 of Kozlov's survey [25], namely, that $\psi(G)$ could also be defined as the minimum $r$ for which $G$ admits a homomorphism into one of the graphs $U(m, r)$. In the language of [25] this defines $\psi(G)$ via the state graphs $U(m, r)$ and valuation $U(m, r) \mapsto r$.

To be able to speak about topological $t$-chromaticity with respect to the graph $U(m, r)$ we need to consider $B_{0}(U(m, r))$. It is going to be useful to introduce an exponentially smaller $\mathbb{Z}_{2}$-equivalent complex.

Definition 4.3. Let $K_{m}$ denote the complete graph on the vertex set $V\left(K_{m}\right)=[m]$ and let $L_{m}=B_{0}\left(K_{m}\right)$. For a positive integer $r \leq m$ let $L_{m, r}$ denote the subcomplex of $L_{m}$ that consists of those simplices $S \uplus T$, for which $|S|<r$ and $|T|<r$. Let $L_{m, r}^{\prime}=L_{m, r} \cup\{S \uplus \emptyset: S \subseteq[m]\} \cup\{\emptyset \uplus T: T \subseteq[m]\}$. The bodies $\left\|L_{m, r}\right\|$ and $\left\|L_{m, r}^{\prime}\right\|$ are $\mathbb{Z}_{2}$-spaces with the involution inherited from $\left\|L_{m}\right\|$.

Lemma 4.4. For every $m$ and $r$ we have $\left\|L_{m, r}^{\prime}\right\| \leftrightarrow\left\|B_{0}(U(m, r))\right\|$.

Proof. We have a simplicial $\mathbb{Z}_{2}$-map $B_{0}(U(m, r)) \rightarrow L_{m, r}^{\prime}$ given by $+(i, A) \mapsto+i$ and $-(i, A) \mapsto-i$. This shows $\left\|B_{0}(U(m, r))\right\| \rightarrow\left\|L_{m, r}^{\prime}\right\|$.

We give a monotonously decreasing map $g$ from the simplices in the barycentric subdivision $s d\left(L_{m, r}^{\prime}\right)$ to the simplices of $B_{0}(U(m, r))$. This map can be considered as a simplicial map from the second subdivision $s d\left(s d\left(L_{m, r}^{\prime}\right)\right)$ to the subdivision $s d\left(B_{0}(U(m, r))\right.$ ) and thus $\|g\|$ (the piecewise linear extension of $g$ ) maps $\left\|s d\left(s d\left(L_{m, r}^{\prime}\right)\right)\right\|=\left\|L_{m, r}^{\prime}\right\|$ to $\left\|s d\left(B_{0}(U(m, r))\right)\right\|=\left\|B_{0}(U(m, r))\right\|$. This is clearly a $\mathbb{Z}_{2}$-map showing $\left\|L_{m, r}^{\prime}\right\| \rightarrow\left\|B_{0}(U(m, r))\right\|$ as stated.

Recall that the vertices of $s d\left(L_{m, r}^{\prime}\right)$ are the simplices of $L_{m, r}^{\prime}$, and a nonempty set of vertices forms a simplex in $s d\left(L_{m, r}^{\prime}\right)$ if it is linearly ordered by inclusion. Therefore let $C$ be a simplex of $s d\left(L_{m, r}^{\prime}\right)$ and let $S \uplus T$ be its smallest vertex and $S^{\prime} \uplus T^{\prime}$ be its largest vertex. We set $g(C)=W \uplus Z$ with $W=\{(i, H) \in V(U(m, r))$ : $\left.i \in S, T^{\prime} \subseteq H\right\}$ and $Z=\left\{(i, H) \in V(U(m, r)): i \in T, S^{\prime} \subseteq H\right\}$. Any pair of vertices $w \in W$ and $z \in Z$ is connected in $U(m, r)$, so $g(C) \in B_{0}(U(m, r))$. The map $g$ is clearly monotonically decreasing. Thus simplices of $s d\left(s d\left(L_{m, r}^{\prime}\right)\right)$ are mapped into simplices of $s d\left(B_{0}(U(m, r))\right)$ provided $g(C)$ is not empty. Assume first that $S \neq \emptyset$. We have $S \subseteq S^{\prime} \neq \emptyset$, so by the definition of $L_{m, r}^{\prime}$ we have $\left|T^{\prime}\right| \leq r-1$. We choose $i \in S$ and a set $H \supseteq T^{\prime}$ with $|H|=r-1$ and $i \notin H$. We have $(i, H) \in W$, so $W \neq \emptyset$. The same argument shows that if $T \neq \emptyset$, then $Z \neq \emptyset$. As $S \uplus T$ is a simplex either $S \neq \emptyset$ or $T \neq \emptyset$, and we have $g(C) \neq \emptyset$ in either case.

Remark 4.5. Though we need only the above proven $\mathbb{Z}_{2}$-equivalence of $\left\|L_{m, r}^{\prime}\right\|$ and $\left\|B_{0}(U(m, r))\right\|$, we mention that they are actually $\mathbb{Z}_{2}$-homotopy equivalent.

To prove this we show that the $\mathbb{Z}_{2}$-mappings $\|f\|:\left\|B_{0}(U(m, r))\right\| \rightarrow\left\|L_{m, r}^{\prime}\right\|$ and $\|g\|:\left\|L_{m, r}^{\prime}\right\| \rightarrow\left\|B_{0}(U(m, r))\right\|$ satisfy that both $\|f\| \circ\|g\|$ and $\|g\| \circ\|f\|$ are $\mathbb{Z}_{2}$-homotopic to the identity of the respective spaces. Here $\mathbb{Z}_{2}$-homotopic means that they are homotopic with every layer of the homotopy being a $\mathbb{Z}_{2}$-map and $f$ is the simplicial map corresponding to the natural coloring of $U(m, r)$, while $g$ denotes the same map as in the proof above. 
Let $a$ and $b$ be $\mathbb{Z}_{2}$-maps from $X$ to $\|C\|$, where $C$ is a simplicial $\mathbb{Z}_{2}$-complex. If for all $x \in X$ the points $a(x)$ and $b(x)$ are contained in the body of a common simplex, then linear interpolation between $a$ and $b$ proves that they are $\mathbb{Z}_{2}$-homotopic.

This simple observation can be directly used to show that $\|f\| \circ\|g\|$ is $\mathbb{Z}_{2^{-}}$ homotopic with the identity on $\left\|L_{m, r}^{\prime}\right\|$. Unfortunately, the same argument cannot be used directly to show that $\|g\| \circ\|f\|$ is homotopic to the identity on $\left\|B_{0}(U(m, r))\right\|$. We introduce the simplicial map $h: s d\left(s d\left(B_{0}(U(m, r))\right)\right) \rightarrow$ $s d\left(B_{0}(U(m, r))\right)$ mapping a chain of simplices from $B_{0}(U(m, r))$ to its smallest element. Clearly, $\|h\|:\left\|B_{0}(U(m, r))\right\| \rightarrow\left\|B_{0}(U(m, r))\right\|$ is a $\mathbb{Z}_{2}$-map. Now the above elementary argument can be used to show that both the identity and $\|g\| \circ\|f\|$ are $\mathbb{Z}_{2}$-homotopic to $\|h\|$. This shows that they are $\mathbb{Z}_{2}$-homotopic to each other, too.

In the following lemma we use the notion of Bier spheres. For a complex $K$ with $V(K) \subseteq[m],[m] \notin K$, its Bier sphere is defined as

$$
\operatorname{Bier}_{m}(K)=\left\{S \uplus T \in L_{m}: S \in K, \bar{T} \notin K, S \cap T=\emptyset\right\},
$$

where $\bar{T}=[m] \backslash T$ is the complement of $T$. The basic result on Bier spheres is that they are always triangulations of a sphere: $\left\|\operatorname{Bier}_{m}(K)\right\| \cong \mathbb{S}^{m-2}$. For a proof of this result, see, e.g., Theorem 5.6.2 in [30], or [12].

Lemma 4.6. For $r \geq 1$ we have $\left\|L_{2 r-1, r}\right\| \cong \mathbb{S}^{2 r-3}$.

Proof. Observe that $L_{2 r-1, r}$ is just the Bier sphere $\operatorname{Bier}_{2 r-1}(K)$ of the simplicial

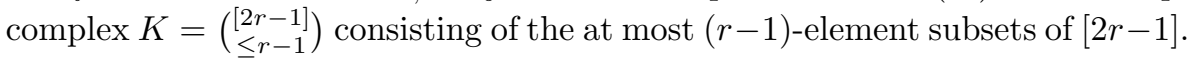

Corollary 4.7. The graph $U(2 r-1, r)$ is topologically $(2 r-2)$-chromatic. In particular we have

$$
\operatorname{coind}\left(\left\|B_{0}(U(2 r-1, r))\right\|\right)=2 r-3 .
$$

Proof. By Lemma 4.4 we have $\operatorname{coind}\left(\left\|B_{0}(U(2 r-1, r))\right\|\right)=\operatorname{coind}\left(\| L_{2 r-1, r}^{\prime}||\right)$. By containment we have $\operatorname{coind}\left(\left\|L_{2 r-1, r}^{\prime}\right\|\right) \geq \operatorname{coind}\left(\left\|L_{2 r-1, r}\right\|\right)$. By Lemma 4.6 (and the remark in the introductory part of Subsection 2.1 about homotopy spheres) we have coind $\left(\left\|L_{2 r-1, r}\right\|\right)=2 r-3$. The reverse inequality follows from applying the inequality $\chi(G) \geq \operatorname{coind}\left(\left\|B_{0}(G)\right\|\right)+1$ to $G=U(2 r-1, r)$ and using the inequality $\chi(U(2 r-1, r)) \leq 2 r-2$. The latter is a special case of the fact mentioned above that $\chi(U(m, r))<m$ if $r<m$.

Remark 4.8. The fact that $\chi(U(2 r-1, r)) \geq 2 r-2$ is a special case of Theorem 2.6 in 14]. This remark parallels Remark 3 in [38, which explains how the upper bound results of [38] imply $\chi(U(2 r, r+1)) \geq 2 r-1$, another special case of Theorem 2.6 in 14. In 38 this follows from the proof of local $(r+1)$-chromaticity of some strongly topologically $(2 r-1)$-chromatic graphs that can be attained by using $2 r$ colors. This implies the existence of homomorphisms from some strongly topologically $(2 r-1)$ chromatic graphs to $U(2 r, r+1)$. Besides implying $\chi(U(2 r, r+1)) \geq 2 r-1$ this also shows that the graphs $U(2 r, r+1)$ are strongly topologically $(2 r-1)$-chromatic. (Their chromatic number is $2 r-1$, indeed, by the same argument we have in the second part of the proof of Corollary 4.7) The above is in contrast to the case of $U(2 r-1, r)$, since these graphs, as stated below in Corollary 4.9, are only topologically $(2 r-2)$-chromatic but not strongly topologically $(2 r-2)$-chromatic. 
Corollary 4.9. For any $r \geq 1$ there exists a topologically $2 r$-chromatic graph with local chromatic number $\psi(G)=r+1$ which can be attained by a $(2 r+1)$ coloring. In particular, topological $2 r$-chromaticity implies neither strong topological $2 r$-chromaticity nor that the local chromatic number is at least $r+2$.

Proof. The example claimed is $U(2 r+1, r+1)$. The local chromatic number is attained by its natural coloring. Topological $2 r$-chromaticity is given by Corollary 4.7. Theorem 3.2 shows that $U(2 r+1, r+1)$ is not strongly topologically $2 r$-chromatic, since then its local chromatic number should be larger.

\section{Direct Separation arguments}

Inequality (2.1) and our statement that $U(2 r+1, r+1)$ satisfies topological $2 r$-chromaticity, but not strong topological $2 r$-chromaticity, show that $H(U(2 r+1, r+1))$ has different index and coindex. While, as we already mentioned, the existence of such spaces has been known (even with arbitrarily high difference between the index and the coindex; see page 100 of 30] and the references therein), $H(U(5,3))$ yields a particularly simple and elementary example. See the argument below on compact orientable 2-manifolds.

First we claim a variant of Lemma 4.4 for the hom space. Let $H_{m, r}=\left\|L_{m, r}\right\| \cap$ $H\left(K_{m}\right)$. We claim that $H(U(m, r)) \leftrightarrow H_{m, r}$. The proof is almost identical to that of Lemma 4.4

Notice that $H_{2 r+1, r+1}$ is a topological $(2 r-2)$-manifold. To see this consider $H_{m, r}$ as the body of the cell complex $\hat{H}_{m, r}=\operatorname{Hom}\left(K_{2}, K_{m}\right) \cap L_{m, r}$. It is enough to verify that it is connected and the link of any vertex is a triangulation of the same sphere. Here the link of a vertex $V$ in the complex $K$ consists of the sets $W \backslash\{V\}$ for cells $W$ in $K$ containing $V$. Note that the link of a vertex in the cell complex $\hat{H}_{m, r}$ is a simplicial complex. By the symmetry of $\hat{H}_{m, r}$, the links of the vertices are isomorphic. The link of the vertex $\{m-1\} \uplus\{m\}$ is $L_{m-2, r-1}$. In the case of $\hat{H}_{2 r+1, r+1}$, this link is $L_{2 r-1, r}$, and thus it is a triangulated $\mathbb{S}^{2 r-3}$ by Lemma 4.6 as needed.

As $H_{2 r+1, r+1}$ is a $(2 r-2)$-manifold embedded in $\left\|L_{2 r+1, r+1}\right\| \cong \mathbb{S}^{2 r-1}$ it is orientable. One can easily compute the Euler characteristic of $H_{5,3}$ directly: its defining cell complex has 20 vertices, 30 cells of dimension 2, and 60 edges; thus the Euler characteristic is -10 . This shows that $H_{5,3}$ is the orientable compact 2 -manifold of genus 6 . Consider this manifold as a sphere $\mathbb{S}^{2}$ with six "handles" arranged in a centrally symmetric manner. The central reflection gives the involution of the space.

The following argument is a direct and simple proof that any compact orientable 2-manifold $T$ of even and positive genus and with the involution as above satisfies that its index is 2 , while its coindex is 1 . We will show that the index is at least 2 by showing that the coindex of its suspension is at least 3 . Thus by the result of Csorba [11] and Živaljević [46] and since these spaces admit triangulations, each of these examples yields different topologically 4-chromatic graphs that are not strongly topologically 4 -chromatic.

Note that $\operatorname{ind}(T) \leq 2$ and $\operatorname{coind}(T) \geq 1$ are trivial.

We prove coind $(\operatorname{susp}(T)) \geq 3$ by giving the explicit mapping. Then ind $(\operatorname{susp}(T))$ $\geq 3$ by the Borsuk-Ulam Theorem and $\operatorname{ind}(T) \geq 2$ follows from Lemma 2.1. We choose $T$ as a subspace of $\mathbb{S}^{3}$ closed for the involution. This can be done in a smooth way such that every point $\boldsymbol{x} \in \mathbb{S}^{3}$ within some distance $\varepsilon>0$ of $T$ has a unique 
closest point $\hat{\boldsymbol{x}} \in T$. We denote by $T^{+}$and $T^{-}$the two components of $\mathbb{S}^{3} \backslash T$. If we identify the points in $T^{+}$far away from $T$ and also identify the points in $T^{-}$far away from $T$, then the resulting factor space is naturally homeomorphic to $\operatorname{susp}(T)$. The resulting map $f: \mathbb{S}^{3} \rightarrow \operatorname{susp}(T)$ can be given as follows:

$$
f(\boldsymbol{x})= \begin{cases}(*, 1) & \text { if } \operatorname{dist}(\boldsymbol{x}, T) \geq \varepsilon, \boldsymbol{x} \in T^{+}, \\ (\hat{\boldsymbol{x}}, \operatorname{dist}(\boldsymbol{x}, T) / \varepsilon) & \text { if } \operatorname{dist}(\boldsymbol{x}, T)<\varepsilon, \boldsymbol{x} \in T^{+}, \\ (\boldsymbol{x}, 0) & \text { if } \boldsymbol{x} \in T, \\ (\hat{\boldsymbol{x}},-\operatorname{dist}(\boldsymbol{x}, T) / \varepsilon) & \text { if } \operatorname{dist}(\boldsymbol{x}, T)<\varepsilon, \boldsymbol{x} \in T^{-} \\ (*,-1) & \text { if } \operatorname{dist}(\boldsymbol{x}, T) \geq \varepsilon, \boldsymbol{x} \in T^{-}\end{cases}
$$

We prove $\operatorname{coind}(T)<2$ by a similar argument as the one hinted in Remark 3.9. A continuous map $f: \mathbb{S}^{2} \rightarrow T$ lifts to the universal covering space $\mathbb{R}^{2}$ of $T$, but by the Borsuk-Ulam Theorem, $\hat{f}: \mathbb{S}^{2} \rightarrow \mathbb{R}^{2}$ identifies two antipodal points of the sphere, so $f$ also identifies two antipodal points, and therefore $f$ is not a $\mathbb{Z}_{2}$-map.

It is worth noting where the argument showing $\operatorname{coind}(\operatorname{susp}(T)) \geq 3$ fails for compact orientable 2-manifolds $T$ of odd genus (with the involution given by the reflection in their standard self-dual embeddings in $\mathbb{S}^{3}$ ). The function $f$ defined as above is not a $\mathbb{Z}_{2}$-map because the involution on $\mathbb{S}^{3}$ does not switch the components of $\mathbb{S}^{3} \backslash T$ in this case. Indeed, we have ind $(T)=1$ for such surfaces $T$.

The argument above shows that for the manifold $T$ considered above one has $\operatorname{coind}(\operatorname{susp}(T))-\operatorname{coind}(T)=2$. It would be interesting to find spaces $T$ with $\operatorname{coind}(\operatorname{susp}(T))-\operatorname{coind}(T)$ arbitrarily large.

The space $T=H_{2 r+1, r+1}$ provides an example of a $\mathbb{Z}_{2}$-space $T$ with $\operatorname{coind}(T) \leq$ $2 r-3$ and $\operatorname{coind}(\operatorname{susp}(T)) \geq 2 r-1$. The following lemma can be used to find examples for spaces $T$ with $\operatorname{coind}(T) \leq d-2$ and $\operatorname{coind}(\operatorname{susp}(T)) \geq d$ also for even values of $d$.

Below we use cohomologies over $\mathbb{Z}_{2}$.

Lemma 5.1. If a compact $d$-manifold $T$ has a nontrivial cohomology $l$ in some dimension $1 \leq i \leq d-1$ and it is the body of a free simplicial $\mathbb{Z}_{2}$-complex, then $\operatorname{coind}(T)<d$.

Proof. We need to show that no $\mathbb{Z}_{2}$-map $f: \mathbb{S}^{d} \rightarrow T$ exists.

Assume for a contradiction that such a map $f$ exists. It induces a reverse map $f^{*}$ on the cohomologies; in particular, it maps $l$ to an $i$-cohomology of $\mathbb{S}^{d}$. As no such nontrivial cohomology exists, we have $f^{*}(l)=0$. By Poincaré duality there exists a $(d-i)$-cohomology $l^{\prime}$ with the cup product $l \smile l^{\prime}=z$ being the only nontrivial $d$-cohomology in $T$. As $f^{*}$ preserves the cup product we have $f^{*}(z)=0$.

As $T$ is the body of a $d$-dimensional free simplicial $\mathbb{Z}_{2}$-complex there is a $\mathbb{Z}_{2}$-map $g: T \rightarrow \mathbb{S}^{d}$. Let $w$ be the only nontrivial $d$-cohomology of $\mathbb{S}^{d}$. The homomorphism $g^{*}$ induced by $g$ maps $w$ either to $z$ or to 0 ; in either case, $(g \circ f)^{*}(w)=f^{*}\left(g^{*}(w)\right)=$ 0 . This shows that $g \circ f: \mathbb{S}^{d} \rightarrow \mathbb{S}^{d}$ has even degree, which contradicts the fact that it is a $\mathbb{Z}_{2}$-map (cf. [10, Theorem 20.6 on page 244). This contradiction proves $\operatorname{coind}(T)<d$.

Let $T$ be a $(d-1)$-manifold obtained by attaching two homeomorphic "handles" to the sphere $\mathbb{S}^{d-1}$. We start with $\mathbb{S}^{d-1} \subset \mathbb{S}^{d}$ and attach the handles inside $\mathbb{S}^{d}$ in a centrally symmetric way and smoothly, just as in the $d=3$ case earlier. The central reflection of $\mathbb{S}^{d}$ gives the involution in $T$. We can prove coind $(\operatorname{susp}(T)) \geq d$ via the same explicit $\mathbb{Z}_{2}$-map $f: \mathbb{S}^{d} \rightarrow \operatorname{susp}(T)$ as in the $d=3$ case. Note that 
the space $T$ constructed admits a triangulation where the involution is simplicial. As $T$ is a $(d-1)$-manifold with the above property, by Lemma 5.1 it is enough to find a nontrivial cohomology (over $\mathbb{Z}_{2}$ ) of $T$ in some dimension $1 \leq i \leq d-2$ and this implies coind $(T) \leq d-2$. By choosing for example handles homeomorphic to a punctured $\mathbb{S}^{k} \times \mathbb{S}^{l}$ with $k, l \geq 1, k+l=d-1$, one makes sure that the $k$ th and $l$ th cohomology groups are nontrivial. More formally, we could say that we construct the manifold $T$ from the sphere $\mathbb{S}^{d-1}$ by applying two $(k-1)$-surgeries $(1 \leq k \leq d-2)$, in a centrally symmetric way.

For every even $t>2$ we have found a graph that is topologically $t$-chromatic but not strongly topologically $t$-chromatic: it is $U(t+1, t / 2+1)$. As we pointed out in Remark 4.8, the analogous graphs $U(t+1,(t+1) / 2+1)$ with $t$ odd are strongly topologically $t$-chromatic and their chromatic number is also $t$. Still, using the $\mathbb{Z}_{2}$-space $T$ constructed in the last paragraph with coind $(T) \leq d-2$ and $\operatorname{coind}(\operatorname{susp}(T)) \geq d$, one can separate topological $t$-chromaticity from strong topological $t$-chromaticity in a similar way also for odd $t$. Choose $d=t-1$. Using again that the space $T$ constructed admits a triangulation where the involution is simplicial we get by the results of Csorba [11] and Živaljević [46] that there exists a finite graph $G$ with $H(G) \mathbb{Z}_{2^{-}}$(homotopy) equivalent to $T$ and therefore $\left\|B_{0}(G)\right\|$ $\mathbb{Z}_{2}$-(homotopy) equivalent to $\operatorname{susp}(T)$. This graph $G$ is topologically $t$-chromatic, but not strongly topologically $t$-chromatic. Thus we proved

Corollary 5.2. For every integer $t>2$ there exists a graph that is topologically t-chromatic, but not strongly topologically t-chromatic.

\section{ACKNOWLEDGMENTS}

We are grateful to Imre Bárány, Péter Csorba, Gábor Elek, László Fehér, László Lovász, Jiří Matoušek, Gábor Moussong, András Szücs, and Rade Živaljević for many clarifying conversations and e-mail messages that improved our understanding of the topological concepts used in this paper.

\section{REFERENCES}

1. J. M. Aarts, R. J. Fokkink, Coincidence and the colouring of maps, Bull. London Math. Soc., 30 (1998), no. 1, 73-79. MR 1479039 (98j:55002)

2. N. Alon, P. Frankl, L. Lovász, The chromatic number of Kneser hypergraphs, Trans. Amer. Math. Soc., 298 (1986), 359-370. MR857448 (88g:05098)

3. D. Archdeacon, J. Hutchinson, A. Nakamoto, S. Negami, K. Ota, Chromatic numbers of quadrangulations on closed surfaces, J. Graph Theory, 37 (2001), no. 2, 100-114. MR.1829924 (2002j:05044)

4. E. Babson, D. N. Kozlov, Complexes of graph homomorphisms, Israel J. Math., 152 (2006), 285-312. MR2214465 (2007b:52024)

5. P. Bacon, Equivalent formulations of the Borsuk-Ulam theorem, Canad. J. Math., 18 (1966), 492-502. MR0195081 (33:3286)

6. I. Bárány, A short proof of Kneser's conjecture J. Combin. Theory Ser. A, 25 (1978), no. 3, 325-326. MR514626 (81g:05056)

7. I. Bárány, personal communication.

8. A. Björner, Topological methods, in: Handbook of Combinatorics (Graham, Grötschel, Lovász eds.), 1819-1872, Elsevier, Amsterdam, 1995. MR.1373690 (96m:52012)

9. A. Björner, M. de Longueville, Neighborhood complexes of stable Kneser graphs, Combinatorica, 23 (2003), no. 1, 23-34. MR.1996625 (2004e:05072)

10. G. Bredon, Topology and Geometry, Graduate Texts in Mathematics 139, Springer-Verlag, New York, 1993. MR1224675 (94d:55001)

11. P. Csorba, Homotopy types of box complexes, Combinatorica, 27 (2007), no. 6, 669-682. 
12. M. de Longueville, Bier spheres and barycentric subdivision, J. Combin. Theory Ser. A, 105 (2004), 355-357. MR2046088 (2005d:52016)

13. P. Erdős, Graph theory and probability, Canad. J. Math., 11 (1959), 34-38. MR0102081 (21:876)

14. P. Erdős, Z. Füredi, A. Hajnal, P. Komjáth, V. Rödl, Á. Seress, Coloring graphs with locally few colors, Discrete Math., 59 (1986), 21-34. MR837951 (87f:05069)

15. K. Fan, A generalization of Tucker's combinatorial lemma with topological applications, $A n-$ nals of Mathematics, 56 (1952), no. 2, 431-437. MR0051506 (14:490c)

16. K. Fan, Evenly distributed subsets of $\mathbb{S}^{n}$ and a combinatorial application, Pacific J. Math., 98 (1982), no. 2, 323-325. MR650012 (84c:54070)

17. L. Fehér, personal communication.

18. C. Godsil, G. Royle, Algebraic Graph Theory, Graduate Texts in Mathematics 207, SpringerVerlag, New York, 2001. MR1829620 (2002f:05002)

19. A. Gyárfás, T. Jensen, M. Stiebitz, On graphs with strongly independent colour-classes, J. Graph Theory, 46 (2004), 1-14. MR2051464(2005e:05047)

20. A. Hatcher, Algebraic Topology, Cambridge University Press, 2002. Electronic version available at http://www.math.cornell.edu/ hatcher/AT/ATpage.html. MR.1867354 (2002k:55001)

21. M. Izydorek, J. Jaworowski, Antipodal coincidence for maps of spheres into complexes, Proc. Amer. Math. Soc., 123 (1995), 1947-1950. MR.1242089 (96c:55002)

22. J. Jaworowski, Existence of antipodal coincidence for maps of spheres, preprint.

23. J. Jaworowski, Periodic coincidence for maps of spheres, Kobe J. Math., 17 (2000), no. 1, 21-26. MR 1801262 (2001k:55007)

24. G. Kalai, personal communication.

25. D. N. Kozlov, Chromatic numbers, morphism complexes, and Stiefel-Whitney characteristic classes. Geometric Combinatorics, 249-315 (E. Miller, V. Reiner, B. Sturmfels eds.) IAS/Park City Mathematics Series 13, American Mathematical Society, Providence, RI; Institute for Advanced Study (IAS), Princeton, NJ, 2007.

26. J. Körner, C. Pilotto, G. Simonyi, Local chromatic number and Sperner capacity, J. Combin. Theory, Ser B., 95 (2005), 101-117. MR2156342 (2006c:05058)

27. I. Křriž, Equivariant cohomology and lower bounds for chromatic numbers, Trans. Amer. Math. Soc., 333 (1992), no. 2, 567-577; I. Kř́iž, A correction to: "Equivariant cohomology and lower bounds for chromatic numbers", Trans. Amer. Math. Soc., 352 (2000), no. 4, 1951-1952. MR:1081939 (92m:05085)

28. L. Lovász, Kneser's conjecture, chromatic number, and homotopy, J. Combin. Theory Ser. A, 25 (1978), no. 3, 319-324. MR.514625 (81g:05059)

29. L. Lovász, Self-dual polytopes and the chromatic number of distance graphs on the sphere, Acta Sci. Math. (Szeged), 45 (1983), 317-323. MR708798(84i:05051)

30. J. Matoušek, Using the Borsuk-Ulam Theorem. Lectures on Topological Methods in Combinatorics and Geometry, Springer-Verlag, Berlin, 2003. MR1988723 (2004i:55001)

31. J. Matoušek, G. M. Ziegler, Topological lower bounds for the chromatic number: A hierarchy, Jahresber. Deutsch. Math.-Verein., 106 (2004), no. 2, 71-90. MR2073516 (2005d:05067)

32. B. Mohar, P. D. Seymour, Coloring locally bipartite graphs on surfaces, J. Combin. Theory Ser. B, 84 (2002), no. 2, 301-310. MR1889261 (2003b:05059)

33. B. Mohar, G. Simonyi, G. Tardos, On the local chromatic number of quadrangulations of surfaces, manuscript in preparation.

34. E. V. Ščepin, A certain problem of L. A. Tumarkin. (Russian) Dokl. Akad. Nauk SSSR, 217 (1974), 42-43; English translation: Soviet Math. Dokl., 15 (1974), no. 4, 1024-1026 (1975). MR 0358764(50:11223)

35. E. R. Scheinerman, D. H. Ullman, Fractional Graph Theory, Wiley-Interscience Series in Discrete Mathematics and Optimization, John Wiley and Sons, Chichester, 1997. MR1481157 (98m:05001)

36. A. Schrijver, Vertex-critical subgraphs of Kneser graphs, Nieuw Arch. Wisk. (3), 26 (1978), no. 3, 454-461. MR.512648 (80g:05037)

37. D. Shkliarsky, On subdivisions of the two-dimensional sphere. (Russian. English summary) Rec. Math. [Mat. Sbornik] N. S. 16(58), (1945), 125-128. MR0013303(7:136d)

38. G. Simonyi, G. Tardos, Local chromatic number, Ky Fan's theorem, and circular colorings, Combinatorica, 26 (2006), 587-626. MR2279672(2007j:05083) 
39. G. Simonyi, G. Tardos, Colorful subgraphs in Kneser-like graphs, European J. Combin., 28 (2007), no. 8, 2188-2200. MR2351519

40. G. Simonyi, G. Tardos, Local chromatic number and topological properties of graphs, DMTCS Proceedings Series, AE (2005), Proceedings of the European Conference on Combinatorics, Graph Theory and Applications, 375-378.

41. M. Stiebitz, Beiträge zur Theorie der färbungskritischen Graphen, Habilitation, TH Ilmenau, 1985.

42. C. Tardif, Fractional chromatic numbers of cones over graphs, J. Graph Theory, 38 (2001), 87-94. MR 1857769 (2002g:05090)

43. A. W. Tucker, Some topological properties of disk and sphere, Proc. First Canadian Math. Congress, Montreal, 1945, University of Toronto Press, Toronto, 1946, 285-309. MR0020254 $(8: 525 \mathrm{~g})$

44. J. W. Walker, From graphs to ortholattices and equivariant maps, J. Combin. Theory Ser. $B, 35$ (1983), 171-192. MR.733022 (86a:05050)

45. D. A. Youngs, 4-chromatic projective graphs, J. Graph Theory, 21 (1996), 219-227. MR 1368748 (96h:05081)

46. R. T. Zivaljević, WI-posets, graph complexes and $\mathbb{Z}_{2}$-equivalences, J. Combin. Theory Ser. A, 111 (2005), no. 2, 204-223. MR2156208(2006e:05191)

47. R. T. Živaljević, personal communication.

Alfréd Rényi Institute of Mathematics, Hungarian Academy of Sciences, 1364 Budapest, POB 127, Hungary

E-mail address: simonyi@renyi.hu

School of Computing Science, Simon Fraser University, Burnaby, British Columbia, Canada V5A 1S6 - And - Alfréd Rényi Institute of Mathematics, Hungarian Academy of Sciences, 1364 Budapest, POB 127, Hungary

E-mail address: tardos@cs.sfu.ca

Faculty of Mathematics, University of Belgrade, Studentski trg 16, P.O.B. 550, 11000 Belgrade, Serbia

E-mail address: vrecica@matf.bg.ac.yu 